\title{
Escala de avaliação da qualidade de vida por meio da Teoria da Resposta ao Item
}

http://dx.doi.org/10.11606/1807-5509201900040495

\author{
Wagner Luiz TESTA* \\ Pablo Magno da SILVEIRA* \\ Adriano Ferreti BORGATTO* \\ Aline Rodrigues BARBOSA*
}

*Universidade Federal de Santa Catarina, Florianópolis, SC, Brasil.

\section{Resumo}

O objetivo deste estudo foi criar uma escala para medir a qualidade de vida (OV) por meio da Teoria da Resposta ao Item (TRI). Para isso, foram coletados dados de 940 profissionais de Educação Física (PEF) registrados no Conselho Regional de Educação Física de Santa Catarina (CREF3-SC) em 2012, por meio do questionário WHOQOL-Bref. Para o tratamento dos dados, foi utilizado os recursos da TRI, com base no modelo de resposta gradual (MRG). Os resultados possibilitaram a criação de uma escala com 26 itens, com quatro categorias ordinais, em cinco níveis da escala. Na amostra dos PEF, os itens mais comuns na QV foram aqueles associados à dor física, à necessidade de tratamento médico e à capacidade de locomoção. Os itens mais incomuns foram aqueles associados às questões do ambiente físico, acesso aos serviços de saúde e questão financeira. 0 WHOQOL-Bref e o MRG mostraram-se adequados e forneceram recursos necessários à construção da escala de qualidade de vida.

Palavras-Chave: Avaliação de Programas e Instrumentos de Pesquisa; Professores; Modelo Ordinal.

\section{Introdução}

Nos dias atuais não existe consenso quanto ao conceito e abordagem de análise da qualidade de vida, embora a preocupação com esta variável venha sendo destacada em diversos estudos ${ }^{1-5}$. De acordo com a Organização Mundial de Saúde $(\mathrm{OMS})^{6}$ a qualidade de vida $(\mathrm{QV})$ pode ser conceituada como a percepção do indivíduo em relação às influências culturais, sociais, políticas e econômicas no contexto de sua vida, que promove o alcance de seus objetivos, projetos e expectativas, conferindo-lhe oportunidades de escolhas. Este conceito abrange a multidimensionalidade dos aspectos relacionados à $\mathrm{QV}$, reconhecidos nos diferentes domínios do instrumento de avaliação proposto pela OMS, para ser aplicado em diferentes culturas ${ }^{7}$.

Pesquisas com diferentes grupos, tais como estudantes de graduação ${ }^{2}$, enfermeiros ${ }^{8}$, trabalhadores da indústria ${ }^{9}$, professores ${ }^{2,4,5}$ e professores de Educação física ${ }^{10,11}$ tem procurado mostrar como as condições de vida afetam a percepção da QV ao mesmo tempo que verificam o quanto da $\mathrm{QV}$ pode ser atribuído às condições externas ${ }^{12}$.

A forma mais comum de avaliação da QV é usando-se questionários ${ }^{1,7}$, sendo o questionário WHOQOL, nas suas diferentes versões, um dos mais utilizados $^{4,8,9}$. $\mathrm{Na}$ avaliação com uso de questionários os resultados são, geralmente, analisados por meio de pontuação simples, de acordo com as marcações dadas a cada item, da chamada Teoria Clássica dos Testes. Esse sistema fica dependente do instrumento escolhido, pois considera a qualidade geral do instrumento, ou seja, uma medida só terá qualidade se o instrumento também tiver qualidade. De forma geral, um indivíduo respondendo a instrumentos semelhantes para avaliar a mesma medida, pode apresentar escores diferentes e consequentemente pode ser classificado em níveis de QV diferentes ${ }^{13}$.

Para aprimorar e qualificar este tipo de avaliação surge a Teoria da Resposta ao Item (TRI), um conjunto de modelos estatísticos que permite mensurar um traço latente, por meio de um conjunto de respostas a itens de um instrumento, 
onde o elemento principal é o item e não o instrumento ${ }^{14}$. Os modelos estatísticos usados pela TRI são baseados na probabilidade de resposta do indivíduo condicionada ao traço latente e as características dos instrumentos de medida ${ }^{15}$. O termo traço latente se refere a traços não observáveis diretamente, tais como, aptidões, grau de satisfação, nível de estresse e $\mathrm{QV}^{16}$.

Portanto, o objetivo deste estudo foi criar uma escala para medir a qualidade de vida a partir de uma amostra de profissionais de Educação Física, por meio da Teoria da Resposta ao Item.

\section{Método}

Trata-se de estudo quantitativo, transversal, do tipo exploratório. Os dados fazem parte da pesquisa intitulada "Condiçõos de Saúde dos profissionais de Educação Física do estado de SC", coordenada por docentes do Centro de Desportos, da Universidade Federal de Santa Catarina (UFSC). Esta pesquisa atendeu aos critérios éticos da Resolução 466/2012 do Conselho Nacional de Saúde, sendo aprovada pelo Comitê de Ética em Pesquisas com seres humanos da UFSC (parecer $n^{\circ}$ 301.490; CAAE 14001813.5.0000.0121).

A população de referência foram os profissionais de Educação Física (PEF), com registro no Conselho Regional de Educação Física de Santa Catarina (CREF3-SC), até dezembro de 2012. O levantamento/listagem dos PEF foi obtido mediante contato com a Presidência do CREF-3/SC. Todos os PEF de SC com registro no CREF ( $\mathrm{n}=13.908)$ foram convidados a responder o questionário e 940 responderam ao mesmo.

Para a coleta dos dados foi criado um endereço eletrônico (e-mail) específico para a realização da pesquisa, de modo a preservar a identidade dos envolvidos, além de facilitar a comunicação entre pesquisadores e participantes.

A partir da lista fornecida pelo CREF-SC os indivíduos foram contatados por meio de seus endereços eletrônicos (e-mails). O e-mail enviado aos PEF apresentava a expressão "pesquisa UFSC” no campo assunto e continha uma carta de apresentação com as informações em relação à procedência dos pesquisadores e o objetivo da pesquisa, além do link para acesso ao questionário. A primeira página do questionário apresentava o termo de consentimento livre e esclarecido com as opções de concordo e não concordo em participar. Ao clicar no item concordo, o indivíduo dava, automaticamente, continuidade ao questionário. Além disso, o termo de consentimento foi anexado à mensagem e pedido aos participantes para inserirem a assinatura digital e devolver o documento. Para esse procedimento de coleta de dados foi utilizado os mecanismos do google docs.

Para medir a QV foi utilizado o instrumento WHOQOL-Bref. Este questionário é formado por 26 questôes (itens), sendo duas questôes gerais da QV (I1 e I2) e as demais referentes aos domínios específicos da QV (físico, psicológico, relações sociais e meio ambiente), proposto pelo grupo de qualidade de vida da Organização Mundial da Saúde ${ }^{6,7}$.

Cada opção de resposta dos itens do instrumento possui 5 categorias, que medem o grau de concordância em escala ordinal. As categorias se diferenciam conforme o tipo de item e também quanto ao domínio no qual o mesmo está associado. Por exemplo, o primeiro item possui as seguintes categorias: muito ruim, ruim, nem ruim nem boa, boa e muito boa. Nas questões de 3 à 9 , as categorias são: nada, muito, pouco, mais ou, menos, bastante e extremamente.

Para posicionar os respondentes e os itens com respostas graduadas nesta escala foi necessário utilizar um modelo probabilístico (EQUAÇÃO 1), sendo utilizado o modelo de resposta gradual, proposto por SAMEJIMA ${ }^{17}$. Nesse modelo, assume-se que as categorias de respostas de um item podem ser ordenadas entre si. Com isso, a probabilidade de um indivíduo $j$ escolher uma categoria $k(\mathrm{k}=1$, $2,3,4,5)$ para um determinado item $i$ é dada por:

$$
\mathrm{P}_{\mathrm{i}, \mathrm{k}}^{+}(\theta)=\frac{1}{1+\mathrm{e}^{-\mathrm{a}_{\mathrm{i}}\left(\theta_{\mathrm{j}}-\mathrm{b}_{\mathrm{i}, \mathrm{k}}\right)}}
$$

EQUAÇÃO 1 - Modelo de resposta gradual. 
$\mathrm{Na}$ qual um item que tem 5 categorias, como os do WHOQOL-Bref, apresenta 4 valores do parâmetro de dificuldade " $b_{k}$ " que deverão ser estimados $\left(b_{2}, b_{3}, b_{4}, b_{5}\right)$, com a restrição de que $\mathrm{b}_{2}<\mathrm{b}_{3}<\mathrm{b}_{4}<\mathrm{b}_{5}$, além do parâmetro de discriminação do item "a" e o valor do traço latente $(\theta)$ de cada respondente. Então, o parâmetro " $b_{k}$ ” irá referenciar ao posicionamento da categoria do item; já, o parâmetro "a" irá demonstrar o quão informativo é cada item em relação à medida $\mathrm{da}$ QV. O parâmetro " $\theta$ " representa o valor da QV.

De maneira geral, os parâmetros dos itens e o traço latente do indivíduo é que determinarão a probabilidade da resposta a uma determinada categoria para um dado item. Estas probabilidades poderão ser melhor identificadas por meio da curva característica do item (CCI) que apresentará simultaneamente todas as curvas correspondentes à probabilidade de resposta a cada uma das categorias ${ }^{13}$. Na etapa 1 foi realizada a estimação dos parâmetros $\left(a, b_{2}\right.$, $\mathrm{b}_{3}, \mathrm{~b}_{4}, \mathrm{~b}_{5}$ ) e seus respectivos erros padrões, que representam o erro de estimativa do parâmetro. $\mathrm{Na}$ etapa 2, fase de estimação do traço latente “ $\theta$ ". Dessa forma, após esse procedimento é que se iniciou o processo de criação da escala.

Para criar a escala de QV, foi necessário atribuir valores comparáveis aos itens e aos

\section{Resultados}

Participaram do estudo 940 indivíduos, sendo importante ressaltar que os dados da amostra coletada foram exclusivamente para calibrar os itens e construir a E-QV, sendo possível a aplicação da escala em outras populações. Para que a calibração dos itens seja eficaz, é necessário que os respondentes apresentem diferentes níveis de QV.

A estimação dos parâmetros, ou calibração dos itens, foi a primeira etapa para a construção da escala. Na TABELA 1 estão representadas as estimativas dos parâmetros "a", " $b_{2}$ ", " $b_{3}$ ", " $b_{4}$ " e " $b_{5}$ ” e seus respectivos erros padrôes (ep). Nesta primeira etapa foram estimados 130 parâmetros, cinco para cada item $\left(a, b_{2}\right.$, $\mathrm{b}_{3}, \mathrm{~b}_{4}, \mathrm{~b}_{5}$ ). Este software utilizou o método da máxima verossimilhança marginal para estimar os parâmetros dos itens. respondentes, para que eles fossem posicionados na mesma escala de medida. Esse posicionamento, denominado ancoragem, envolve a estimação dos parâmetros e o cálculo das probabilidades. Já os respondentes são posicionados pela medida do traço latente.

Desse modo, devido à natureza arbitrária dos valores para posicioná-los na escala é criado um valor de referência onde a média foi zero e o desvio padrão um, então dizemos que foi criada uma escala $(0,1)$ que não tem significado prático, ou seja, esta escala poderia ter qualquer valor de referência.

Para que estes valores arbitrários tenham significados, quando os itens são posicionados na escala, o conteúdo abordado em cada item faz com que estes valores tenham interpretação para o traço latente ${ }^{14}$.

Para posicionar os respondentes e os itens com respostas ordinais nesta escala, foi necessário utilizar um modelo matemático, que tem como base a probabilidade. Em modelos com resposta dicotômica, o item fica posicionado somente em um ponto da escala; porém, no modelo probabilístico de resposta gradual (MRG) ${ }^{17}$, cada categoria do item é posicionada na escala, consequentemente um item fica posicionado em mais de um ponto da escala.

Diante disso, nos itens 7, 13 e 15, a categoria que é representada pelo parâmetro " $b_{2}$ " foi agrupada com o parâmetro " $b_{3}$ " por apresentar poucas respostas. Outra questão importante foi o fato da inversão das categorias dos itens: 3,4 e 26. Na primeira rodagem não foi considerada essa inversão, visto que os parâmetros "a" foram muito baixos. Isso ocorreu devido ao fato do instrumento apresentá-las no sentido contrário ao da lógica da escala da QV, o que não faria sentido para a análise dos dados e muito menos para construção e interpretação da E-QV. Na prática, estes itens tiveram suas categorias invertidas. Ou seja, a primeira categoria de resposta (1) foi associada ao parâmetro " $b_{2}$ " e foi a maior categoria, diferentemente dos outros itens, nos quais a maior categoria (5) foi associada ao último parâmetro $\left(b_{5}\right)$. 
TABELA 1 -Estimativas dos parâmetros e dos erros padrões (ep) dos itens para cada categoria de resposta e seus respectivos erros da segunda rodagem

\begin{tabular}{|c|c|c|c|c|c|c|c|c|c|c|}
\hline ITEM & a & ep(a) & $b_{2}$ & $\operatorname{ep}\left(b_{2}\right)$ & $b_{3}$ & $\operatorname{ep}\left(b_{3}\right)$ & $\mathrm{b}_{4}$ & $\operatorname{ep}\left(b_{4}\right)$ & $b_{5}$ & $\operatorname{ep}\left(b_{5}\right)$ \\
\hline 1 & 1,96 & 0.14 & $-3,86$ & 0.45 & $-2,30$ & 0.15 & $-0,91$ & 0.07 & 1,46 & 0.09 \\
\hline 2 & 1,53 & 0.11 & $-3,22$ & 0.34 & $-1,79$ & 0.14 & $-0,80$ & 0.08 & 1,67 & 0.12 \\
\hline 3 & 1,12 & 0.10 & $-4,78$ & 0.55 & $-2,61$ & 0.24 & $-1,22$ & 0.13 & 0,27 & 0.09 \\
\hline 4 & 1,08 & 0.10 & $-4,47$ & 0.51 & $-2,71$ & 0.26 & $-1,63$ & 0.17 & 0,08 & 0.09 \\
\hline 5 & 1,53 & 0.12 & $-4,14$ & 0.47 & $-1,90$ & 0.14 & $-0,16$ & 0.07 & 2,47 & 0.19 \\
\hline 6 & 1,21 & 0.10 & $-5,10$ & 0.68 & $-3,63$ & 0.34 & $-1,84$ & 0.16 & 0,66 & 0.09 \\
\hline 7 & 1,24 & 0.11 & & & $-2,61$ & 0.22 & $-0,53$ & 0.09 & 2,54 & 0.22 \\
\hline 8 & 1,70 & 0.12 & $-4,17$ & 0.51 & $-2,13$ & 0.14 & $-0,41$ & 0.07 & 1,86 & 0.12 \\
\hline 9 & 1,07 & 0.10 & $-4,48$ & 0.50 & $-2,19$ & 0.21 & 0,31 & 0.09 & 3,01 & 0.28 \\
\hline 10 & 2,04 & 0.13 & $-3,64$ & 0.38 & $-2,18$ & 0.14 & $-0,07$ & 0.06 & 1,31 & 0.08 \\
\hline 11 & 1,27 & 0.10 & $-4,44$ & 0.50 & $-2,84$ & 0.23 & $-0,90$ & 0.10 & 0,77 & 0.10 \\
\hline 12 & 1,21 & 0.10 & $-3,60$ & 0.33 & $-1,51$ & 0.13 & 1,09 & 0.11 & 2,59 & 0.22 \\
\hline 13 & 1,11 & 0.09 & & & $-3,36$ & 0.31 & $-0,65$ & 0.10 & 1,83 & 0.17 \\
\hline 14 & 1,30 & 0.10 & $-3,70$ & 0.33 & $-1,29$ & 0.12 & 0,55 & 0.08 & 2,26 & 0.18 \\
\hline 15 & 1,43 & 0.12 & & & $-3,43$ & 0.33 & $-2,05$ & 0.16 & 0,15 & 0.08 \\
\hline 16 & 1,31 & 0.10 & $-3,38$ & 0.29 & $-1,25$ & 0.12 & $-0,23$ & 0.08 & 1,70 & 0.14 \\
\hline 17 & 2,50 & 0.16 & $-3,42$ & 0.34 & $-1,75$ & 0.09 & $-0,75$ & 0.06 & 1,10 & 0.07 \\
\hline 18 & 2,33 & 0.15 & $-3,00$ & 0.23 & $-1,75$ & 0.10 & $-0,82$ & 0.06 & 1,17 & 0.07 \\
\hline 19 & 2,28 & 0.14 & $-3,22$ & 0.28 & $-1,83$ & 0.10 & $-0,73$ & 0.06 & 1,27 & 0.07 \\
\hline 20 & 1,22 & 0.10 & $-4,37$ & 0.46 & $-2,66$ & 0.22 & $-0,99$ & 0.11 & 1,66 & 0.15 \\
\hline 21 & 1,15 & 0.09 & $-4,24$ & 0.45 & $-2,26$ & 0.20 & $-0,92$ & 0.11 & 1,53 & 0.15 \\
\hline 22 & 1,05 & 0.09 & $-5,26$ & 0.64 & $-3,05$ & 0.28 & $-0,74$ & 0.28 & 1,99 & 0.19 \\
\hline 23 & 0,87 & 0.09 & $-5,56$ & 0.71 & $-3,43$ & 0.38 & $-1,73$ & 0.20 & 1,12 & 0.16 \\
\hline 24 & 0,76 & 0.09 & $-4,20$ & 0.50 & $-1,49$ & 0.50 & 0,33 & 0.13 & 3,46 & 0.13 \\
\hline 25 & 0,73 & 0.08 & $-4,93$ & 0.63 & $-2,74$ & 0.34 & $-1,39$ & 0.20 & 1,78 & 0.24 \\
\hline 26 & 1,29 & 0.11 & $-3,73$ & 0.37 & $-2,48$ & 0.20 & $-1,36$ & 0.12 & 1,93 & 0.16 \\
\hline
\end{tabular}

Observa-se na TABELA 1, que os valores dos parâmetros de dificuldade "b", obtiveram valores negativos e positivos. Espera-se que as categorias de um item estejam posicionadas em diferentes pontos da escala, mostrando que esse item possui alto poder discriminatório, ou seja, consegue medir tanto indivíduos com alta probabilidade de responder o item de forma mais positiva como os de responder de forma mais negativa.

Tendo como referência a categoria " $\mathrm{b}_{5}$ ”, os valores do parâmetro "b" variaram de 0,08 a 3,46. Os itens com os menores valores, foram considerados os mais fáceis dos respondentes contemplarem de forma positiva na sua $\mathrm{QV}$, respectivamente: 3, 4 e 15 . Os itens que apresentaram os maiores valores, foram considerados os mais difíceis de serem contemplados de forma positiva pelos respondentes, em ordem crescente: 12, 9 e 24.

Os resultados mostraram que todos os itens do instrumento foram eficientes para medir a QV. De acordo com os dados da TABELA 1 o parâmetro de discriminação "a" variou de 0,73 a 2,50. Valores do parâmetro $\mathrm{a} \geq 0,70$ indicam que os itens possuem bom poder de discriminação e que estão associados ao traço latente que se está medindo ${ }^{15}$. Os três itens com maior grau 
de discriminação foram, em ordem crescente, os itens 19,18 e 17, enquanto os itens com menor poder de discriminação foram os de número 25 , 23 e 24. Dessa forma, é possível afirmar que o item que melhor avaliou a QV, foi o item $17(\mathrm{a}=$ 2,50 ), que mede a capacidade para desempenhar as atividades do dia-a-dia. $\mathrm{O}$ item com menor poder de discriminação, ou seja, com o menor valor de "a" foi o item $25(\mathrm{a}=0,73)$, que mede a satisfação com o meio de transporte.

Por meio da FIGURA 1, que representa a curva de informação dos itens I17 e I25 foi possível comparar a diferença da quantidade de informação dos mesmos. O item I17, em comparação ao I25 apresentou pico maior de informação e mais espalhado ao longo da escala da QV.
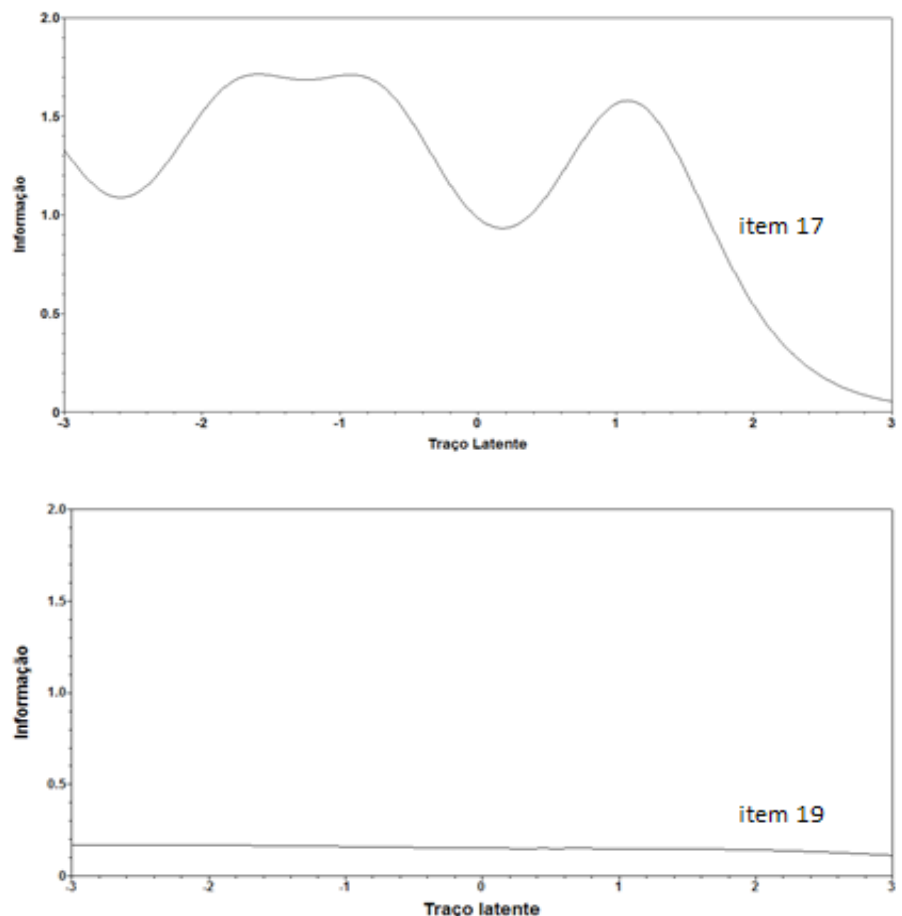

FIGURA 1 -Curvas de informação dos itens 17 e 25.

No MRG, um item com 5 categorias possui 5 curvas características dos itens dadas pela função do próprio modelo e para formar essas cinco curvas são necessários 4 parâmetros de dificuldade (parâmetro $\left.b_{k}\right)\left(b_{2}, b_{3}, b_{4}, b_{5}\right)^{13}$. De acordo com a FIGURA 2 , o parâmetro $b_{2}$ representa o ponto em que a categoria 2 foi mais provável do que a categoria 1; $\mathrm{o}_{3}$ é o ponto em que a categoria 3 se tornou mais provável do que a categoria $2 ; \mathrm{o}_{4}$ é o ponto em que a categoria 4 foi mais provável do que a categoria 3 e o $b_{5}$ o ponto em que a categoria 5 se tornou mais provável do que a categoria 4 .

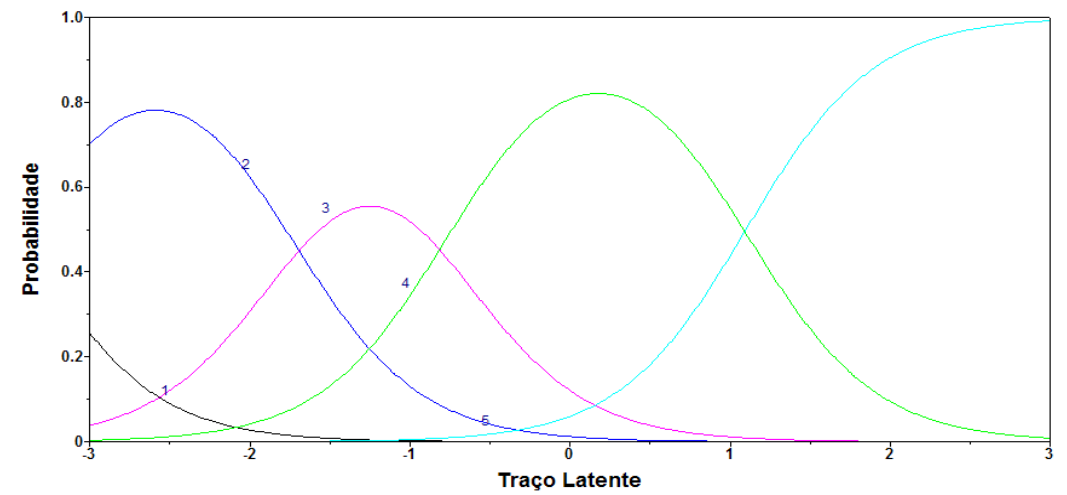

linha 2 - parâmetro $\mathrm{b}_{2}$ (segunda categoria de resposta 2); linha 3 - parâmetro $\mathrm{b}_{3}$ (terceira categoria de resposta 2 ); linha 4 - parâmetro $\mathrm{b}_{4}$ (quarta categoria de resposta 2 ); linha 5 - parâmetro $\mathrm{b}_{5}$ (quinta categoria de resposta 2)

FIGURA 2 -Curva característica do item 17. 
linha contínua: curva do teste / linha pontilhada: erro padrão (ep)
Por meio da curva de informaçáo do teste (FIGURA 3), pode-se observar que o erro padrão (EP) aumenta muito após o valor $2 \mathrm{da}$ escala. Desta forma, este instrumento apresentou informaçóes entre os intervalos de -3 a 2, significando que ele é mais adequado para medir a QV de indivíduos posicionados entre os pontos -3 a 2 da escala ${ }^{14}$.

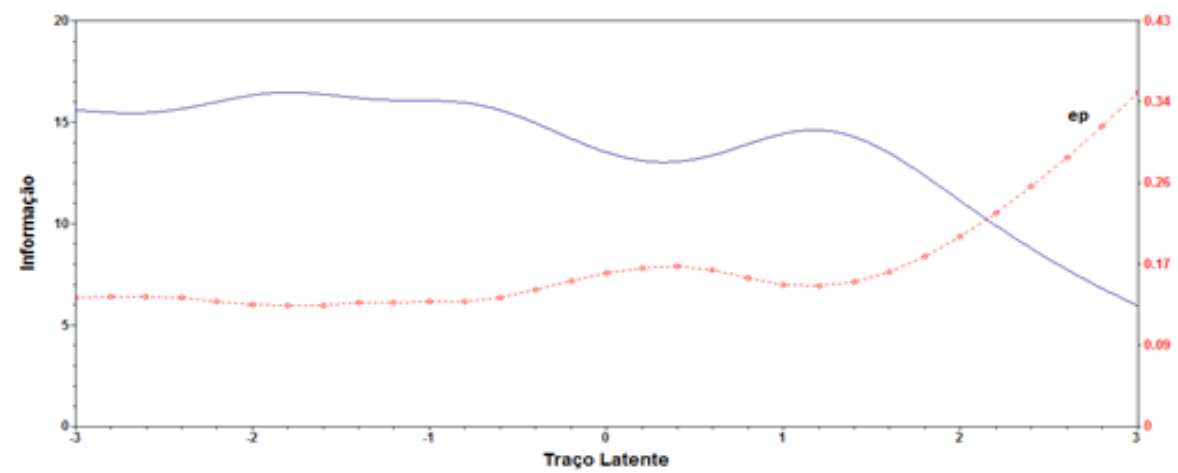

FIGURA 3 -Curva de informação do teste.

Ainda em relação à FIGURA 3, a linha pontilhada, em vermelho, representa o erro padrão. Quanto menor o erro padrão maior será a curva de informação do teste (CIT) em determinado intervalo. Isso foi identificado nas regiôes do nível de QV em que houveram quantidades maiores de informaçóes: de -3 a 2 na escala $(0,1)^{15}$.

A escala foi construída inicialmente na métrica com média 0 (zero) e desvio padrão 1 (um) escala $(0,1)$. Essa métrica foi utilizada por facilitar

$$
\begin{aligned}
& \theta^{*}=10 \times \theta+50 \\
& b_{K}^{*}=10 \times b_{K}+50 \\
& a^{*}=a / 10
\end{aligned}
$$

A escala de QV é uma escala arbitrária em que o importante são as relações de ordem existentes entre seus pontos e não a sua magnitude. Esta escala foi construída com base nos níveis âncoras, obtidos pelo processo de estimação dos parâmetros, assim como pelas probabilidades para os parâmetros " $\mathrm{b}_{2}, \mathrm{~b}_{3}, \mathrm{~b}_{4}, \mathrm{~b}_{5}$ ". Considerou-se que a categoria do o processo computacional para estimação dos parâmetros dos itens. No entanto, a escala sofreu transformações na sua métrica para facilitar o processo de interpretação e passou para a métrica: média 50 e desvio padrão 10 , ou seja, escala $(50,10)$ que pode ser observado no QUADRO 1. Isso foi realizado por meio das equações (2), (3), e (4) que transformaram o nível de QV $(\theta)$ e os parâmetros $\left(a, b_{2}, b_{3}, b_{4}\right.$ e $\left.b_{5}\right)$ da escala $(0,1)$ para a escala $(50,10)$ :

item será posicionada no nível em que $\mathrm{P}(\mathrm{U}=1 \mid$ $\theta) \geq 0,65^{13}$.

Para a construção da escala definiu-se cinco níveis de QV, a saber: muito insatisfeito, insatisfeito, Nem insatisfeito nem satisfeito, satisfeito e muito satisfeito. A descrição de cada um desses níveis

\begin{tabular}{|c|c|c|c|}
\hline Nível & Intervalo & $\%$ & Descrição dos Níveis \\
\hline $\begin{array}{c}\text { Muito } \\
\text { Insatisfeito }\end{array}$ & {$[0-10)$} & $0 \%$ & $\begin{array}{c}\text { Neste nível o respondente está “muito insatisfeito” em com todas as ques- } \\
\text { tões da QV, mas começa a ficar “insatisfeito” com dor física (que o impede de } \\
\text { fazer o que precisa), com algum tratamento médico, com o sentido que a vida } \\
\text { tem, com a sua aparência física, com suas relações pessoais (amigos, parentes, } \\
\text { conhecidos, colegas), com o apoio que recebe de seus amigos e com o seu } \\
\text { meio de transporte. }\end{array}$ \\
\hline
\end{tabular}
estão apresentados no QUADRO 1.

QUADRO 1 - Curva de informação do teste. 
continuação

\begin{tabular}{|c|c|c|c|}
\hline Nível & Intervalo & $\%$ & Descrição dos Níveis \\
\hline Insatisfeito & {$[20-30)$} & $3 \%$ & $\begin{array}{l}\text { Neste nível, o respondente está “insatisfeito” com a sua capacidade de apro- } \\
\text { veitar a vida, com a sensação de segurança em sua vida diária, com a energia } \\
\text { que possui para realizar as tarefas no seu dia-a-dia, com sua condição financei- } \\
\text { ra, com as oportunidades de atividade de lazer, com a qualidade do seu sono, } \\
\text { com sua capacidade de desempenhar as atividades do seu dia-a-dia, com a sua } \\
\text { vida sexual e com o acesso aos serviços de saúde, mas começa a ficar “nem } \\
\text { insatisfeito nem satisfeito” com sua QV, com sua saúde, com dores físicas } \\
\text { (que o impede de fazer o que precisa), com algum tratamento médico para levar } \\
\text { a vida, com o sentido que a vida tem, com sua capacidade de concentração, com } \\
\text { sua aparência física, com a disponibilidade das informações que precisa no seu } \\
\text { dia-a-dia, com sua capacidade de locomoção, com as condições do local onde } \\
\text { mora, com sua capacidade para o trabalho, com suas relações pessoais, com o } \\
\text { apoio que recebe de seus amigos, com o seu meio de transporte e com o aspec- } \\
\text { to emocional, pois ainda apresenta sentimentos negativos. }\end{array}$ \\
\hline $\begin{array}{c}\text { Nem Insatisfei- } \\
\text { to Nem } \\
\text { Satisfeito }\end{array}$ & {$[40-50)$} & $45 \%$ & $\begin{array}{l}\text { Neste nível, o respondente está “nem insatisfeito nem satisfeito" com a } \\
\text { sua capacidade de aproveitar a vida, com a sensação de segurança em sua vida } \\
\text { diária, com a sua condição financeira, com as oportunidades de atividade de } \\
\text { lazer, com o seu sono, com sua capacidade de desempenhar as atividades do seu } \\
\text { dia-a-dia e com o acesso aos serviços de saúde, mas começa a ficar "satisfeito" } \\
\text { com sua QV, com sua saúde, com a questão das dores físicas, com a necessidade } \\
\text { de não fazer tratamento médico, com o sentido que a vida tem, com a sua capa- } \\
\text { cidade de concentração, com a sensação de segurança em sua vida diária, com a } \\
\text { sua aparência física, com a disponibilidade das informações que precisa no seu } \\
\text { dia-a-dia, com a capacidade de locomoção, com sua capacidade de desempenhar } \\
\text { as atividades do seu dia-a-dia, com sua capacidade para o trabalho, consigo } \\
\text { mesmo, com suas relações pessoais, com sua vida sexual, com o apoio que re- } \\
\text { cebe de seus amigos, com as condições do local onde mora, com o seu meio de } \\
\text { transporte e com o aspecto emocional (muito poucos sentimentos negativos) }\end{array}$ \\
\hline Satisfeito & {$[60-70)$} & $50 \%$ & $\begin{array}{l}\text { Neste nível, o respondente está “satisfeito" com as oportunidades de aprovei- } \\
\text { tar a vida, com o seu ambiente físico, com a questão financeira, com as oportu- } \\
\text { nidades de atividade de lazer e com o acesso aos serviços de saúde e começa a } \\
\text { ficar "muito satisfeito" com a sua QV, com a sua saúde; com ausência de dor } \\
\text { (física), com a questão de não precisar de nenhum tratamento médico, com o } \\
\text { sentido que a vida tem, com a energia para realizar as tarefas do dia-a-dia, com a } \\
\text { sua aparência física, com a sua capacidade de locomoção, com o seu sono, com } \\
\text { sua capacidade de desempenhar as atividades do seu dia-a-dia, com sua capaci- } \\
\text { dade para o trabalho, consigo mesmo, com suas relações pessoais, com sua vida } \\
\text { sexual e com as condições do local onde mora. }\end{array}$ \\
\hline $\begin{array}{c}\text { Muito } \\
\text { Satisfeito }\end{array}$ & {$[80-90)$} & $2 \%$ & $\begin{array}{l}\text { Neste nível, o respondente está “muito satisfeito” com a capacidade de apro- } \\
\text { veitar a vida, com a capacidade de concentração, com a sensação de segurança } \\
\text { em sua vida diária, com seu ambiente físico, com a questão financeira, com a } \\
\text { disponibilidade das informações que precisa no seu dia-a-dia, com as oportu- } \\
\text { nidades de atividade de lazer, com o apoio que recebe de seus amigos, com o } \\
\text { acesso de serviços de saúde, com o seu meio de transporte e com o aspecto } \\
\text { emocional (não apresenta sentimentos negativos). }\end{array}$ \\
\hline
\end{tabular}

\section{Discussão}

Devido a característica exploratória e inovadora desta pesquisa, não foi possível comparar os resultados da construção da escala de QV com outros estudos semelhantes. Contudo, de modo a explorar melhor o fenômeno, foi possível comparar com estudos de outras áreas que utilizam a TRI na construção de escalas e com pesquisas específicas que tratam dos itens abordados nesta escala de QV.

Os resultados obtidos pela TRI possibilitaram a diferenciação dos itens mais relevantes da QV, 
fato este, que não poderia ser realizado por meio da Teoria Clássica dos testes. Os parâmetros de cada item são fonte de informação específica em comparação com informações do conjunto dos itens que formam o instrumento ${ }^{14}$ e que, no caso do presente estudo, caracterizam a QV. Desse modo, a TRI permitiu interpretações do todo e também de parte dos resultados, que seriam estudados separadamente. É nesse contexto que a construção desta escala de QV ganha significado prático ${ }^{15}$.

Os itens com maior e menor relevância na $\mathrm{QV}$ dos PEF se referem a características já abordadas, de maneira específica, em outras pesquisas. Desse modo, os itens mais difíceis dos PEF contemplarem em sua QV (I12, I9 e I24) associam, respectivamente a questão ambiental ${ }^{18}$, ao acesso aos serviços de saúde ${ }^{19}$ e à questão financeira ${ }^{20}$. Por outro lado, os itens mais básicos contemplados pelos PEF na sua QV (I3, I4 e I15) referem-se à dor física, a necessidade de tratamento médico para levar a vida e a capacidade de locomoção, respectivamente ${ }^{21}$.

Outro resultado importante encontrado neste trabalho foi a associação entre a saúde e QV (itens gerais do WHOQOL, I1 e I2). Por exemplo, os PEF que estão no nível 60-70 da escala estão muito satisfeitos com sua saúde e com sua QV (50\% dos profissionais). Além disso, estes resultados refletem positivamente quando se realiza a análise dos itens do domínio físico da QV (I3, I4, I10, I15, I16 e I18). Ou seja, a saúde e a QV têm relação direta ${ }^{22,23}$, indicando que para possuir boa saúde é fundamental que se tenha uma boa QV, principalmente no aspecto físico.

Acredita-se que o bom desempenho apresentado pelos PEF nos itens do domínio físico deve-se ao fato das características que a profissão. Ou seja, o dia a dia do PEF é, geralmente, fisicamente ativo. Caminhar, correr, pular, carregar pesos e alongar são atividades rotineiras contribuindo para a melhora e a manutenção da aptidão física ${ }^{24}$.

Outro estudo, com finalidade semelhante a este indica que a TRI é eficaz na construção de escalas para medir a QV. O estudo de $\operatorname{LiN}^{25}$ utilizando os recursos da TRI, por meio do $\mathrm{MRG}^{17}$, examinou as propriedades psicométricas do instrumento WHOQOL-Bref na população de Taiwan. Os autores verificaram que os itens com maior poder discriminação foram: "I22, I20, I18, I16", concordando parcialmente com o presente estudo.

Os itens do WHOQOL-Bref apresentaram boa capacidade de discriminação para medir a QV. Os itens mais fáceis de serem respondidos de forma positiva pelos $\mathrm{PEF}$ em relação a sua $\mathrm{QV}$ foram aqueles associados à dor física (I3), à necessidade de tratamento médico (I4) e à capacidade de locomoção (I15). Por outro lado, os itens mais difíceis de serem respondidos de forma positiva foram aqueles associados às questóes do ambiente físico (I9), ao acesso aos serviços de saúde (I24) e à questão financeira (I12).

$\mathrm{O}$ instrumento WHOQOL-Bref é um instrumento indicado para investigar a $\mathrm{QV}$ e, assim como o MRG mostrou-se adequado e forneceu recursos necessários para a construção da escala de QV. A escala criada foi composta por 5 níveis, com a maior parte dos itens concentrados na parte central da escala.

Portanto, por meio da escala de QV é possível realizar inferências qualitativas e quantitativas dentro do contexto da $\mathrm{QV}$, pois ao mesmo tempo pode-se ordenar os itens e os respondentes na mesma métrica. Cabe salientar, que o instrumento WHOQOL-Bref é um instrumento consolidado na literatura criado pelo grupo de qualidade de vida da Organização Mundial de Saúde e a criação dessa escala contou com a aplicação desse instrumento a uma amostra de profissionais de Educação Física do estado de Santa Catarina/Brasil. Esta escala, bem como sua interpretação, não se limita a essa amostra dos PEF e permite que outras populações sejam avaliadas por ela, bastando usar os parâmetros gerados por este estudo.

Como sugestão para futuros estudos, fica a aplicação dessa escala em outras populações, a fim de confrontar os resultados e novas pesquisas que façam avançar os resultados encontrados neste estudo.

\section{Agradecimentos}

Os autores agradecem ao Conselho Regional de Educação Física, de Santa Catarina; ao Prof. Marino Tessari, Prof. Dr. Juarez Vieira do Nascimento e aos participantes do estudo. 


\begin{abstract}
Quality assessment scale through item response theory

The purpose of this study was create a scale to measure the quality of life (OL) through Item Response Theory (IRT). For this, data were collected from 940 Physical Education professionals (PEP) registered the Regional Council of Physical Education of Santa Catarina (CREF3-SC) in 2012, through the WHOOOL-Bref questionnaire. Data analysis was done by TRI, using the graduated response model (GRM). The results enabled the creation of a scale with 26 items, four ordinal categories, into five levels of scale. In the sample of the PEP, the most common items on $\mathrm{QL}$ were those associated with physical pain, the need for medical treatment and walking ability. The most unusual items were those associated with the issues of the physical environment, access to health services and financial. The WHOOOL-Bref and GRM proved adequate and provide resources necessary to build the quality of life scale.
\end{abstract}

KEYwoRDS: Program Evaluation and Research Tools; Faculty; Ordinal Model.

\title{
Referências
}

1. Campolina AG, Bortoluzzo AB, Ferraz MB, Ciconelli RM. Validação da versão brasileira do questionário genérico de qualidade de vida short-form 6 dimensions (SF-6D Brasil) Validation of the brazilian version of the generic sixdimensional short form quality of life questionnaire (SF-6D Brazil). Cien Saude Colet. 2011;16(7):3103-10.

2. Samulski DM, Noce F. A importância da atividade física para a saúde e qualidade de vida: um estudo entre professores, alunos e funcionários da UFMG. Rev bras ativ fís saúde. 2012;5(1):5-21.

3. Pereira ÉF, Teixeira CS, dos Santos A. Qualidade de vida: abordagens, conceitos e avaliação. Rev Bras Educ Fís Esporte. 2012;26(2):241-50.

4. Pereira E, Teixeira CS, Lopes AS. Qualidade de vida de professores de educação básica do município de Florianópolis, SC, Brasil. Cien Saude Colet. 2013;18(7):1963-70.

5. Catapan A, Bonfim BLS, Panucci-Filho L, de Oliveira EG, Vila EW, dos Reis EB. Rev Bras Qual Vida. 2014.

6. The World Health Organization quality of life assessment position paper from the World Health Organization. Soc Sci Med. 1995;41(10):1403-9.

7. Fleck MP, Louzada S, Xavier M, Chachamovich E, Vieira G, Santos L, et al. Aplicação da versão em português do instrumento abreviado de avaliação da qualidade de vida "WHOQOL-bref”. Rev saúde pública. 2000;34(2):178-83.

8. Fernandes JS, Miranzi SdSC, Iwamoto HH, dos Santos Tavares DM, Dos Santos CB. A relação dos aspectos profissionais na qualidade de vida dos enfermeiros das equipes Saúde da Família. Rev Esc Enferm USP. 2012;46(2):404-12.

9. Costa CSN, Freitas EG, Souza Mendonça LC, Alem MER, Coury HJCG. Capacidade para o trabalho e qualidade de vida de trabalhadores industriais Work ability and quality of life of Brazilian industrial workers. Ciênc Saúde Coletiva. 2012;17(6):1635-1642.

10. Both J, Nascimento JV, Borgatto AF. Percepção da qualidade de vida no trabalho ao longo da carreira docente. Rev bras cineantropom desempenho hum. 2008;10(4):372-8.

11. Moreira HR, Farias GO, Both J, Nascimento JV. Qualidade de vida no trabalho e síndrome de burnout em professores de educação física do estado do Rio Grande do Sul, Brasil. Rev bras ativ fís saúde. 2012;14(2):115-22.

12. Andrews FM, Withey SB. Social indicators of well-being: Americans' perceptions of life quality. Plenum Press: Springer US; 2012.

13. Andrade DF, Tavares HR, Valle RC. Teoria da Resposta ao Item: Conceitos e Aplicaçóes. 14 Simpósio Nacional de Probabilidade e Estatística; 2000 jul 28; Caxambu. São Paulo (SP): Associação Brasileira de Estatística; 2000. p.164.

14. Araujo EAC, Andrade DF, Bortolotti SLV. Teoria da Resposta ao Item. Rev Esc Enferm USP. 2009;43:1000-8.

15. Bortolotti SLV, Tezza R, Andrade DF, Bornia AC, Sousa Júnior AF. Relevance and advantages of using the item response theory. Qual Quant. 2013;47(4):2341-60.

16. Pasquali L, Primi R. Fundamentos da teoria da resposta ao item: TRI. Aval Psicol. 2003;2(2):99-110.

17. Samejima FA. Estimation of latent ability using a response pattern of graded scores. Psychometrika. 1969;34(suppl 
1):1-97.

18. Gordia AP, Quadros TMB, Campos W. Variáveis sociodemográficas como determinantes do domínio meio ambiente da qualidade de vida de adolescentes. Ciên Saude Colet. 2009;14(16):2261-8.

19. Minayo MCS, Hartz ZMA, Buss PM. Qualidade de vida e saúde: um debate necessário. Cien Saude Colet. 2000;5(1):718.

20. Abreu N, Baracho E, Tirado M, Dias R. Qualidade de vida na perspectiva de idosas com incontinência urinária. Rev Bras Fisioter. 2007;11(6):429-36.

21. Corbacho MI, Dapueto JJ. Avaliação da capacidade funcional e da qualidade de vida de pacientes com artrite reumatoide. Rev Bras Reumatol. 2010;50(1):31-43.

22. Silva RS, Silva I, Silva RA, Souza L, Tomasi E. Atividade física e qualidade de vida. Cien Saude Colet. 2010;15:115-20.

23. Pucci GCMF, Rech CR, Fermino RC, Reis RS. Associação entre atividade física e qualidade de vida em adultos. Rev Saude Publica. 2012;46(1):166-79.

24. Valério L. Qualidade de vida e nível de atividade física de profissionais de educação física da rede estadual de ensino de Santa Catarina. Ágora. 2012;17(2):81-93.

25. Lin TH. Identifying optimal items in quality of life assessment. Qual Quant. 2007;41(5):661-72.

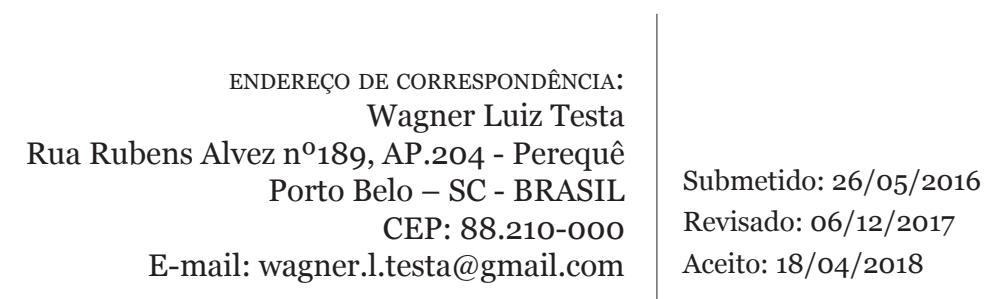

